Article

\title{
Business Cycle Estimation with High-Pass and Band-Pass Local Polynomial Regression
}

\author{
Luis J. Álvarez \\ Banco de España, Madrid 28014, Spain; ljalv@bde.es; Tel.: +34-91-338-5042 \\ Academic Editors: Gilles Dufrénot, Fredj Jawadi and Alexander Mihailov \\ Received: 30 June 2016; Accepted: 16 December 2016; Published: 5 January 2017
}

\begin{abstract}
Filters constructed on the basis of standard local polynomial regression (LPR) methods have been used in the literature to estimate the business cycle. We provide a frequency domain interpretation of the contrast filter obtained by the difference of a series and its long-run LPR component and show that it operates as a kind of high-pass filter, so that it provides a noisy estimate of the cycle. We alternatively propose band-pass local polynomial regression methods aimed at isolating the cyclical component. Results are compared to standard high-pass and band-pass filters. Procedures are illustrated using the US GDP series.
\end{abstract}

Keywords: business cycles; local polynomial regression; filtering; high-pass; band-pass; US cycles

\section{JEL Classification: C13}

\section{Introduction}

There is a large body of literature on methods to estimate the business cycle [1-4] and the Great Recession has rekindled the interest in business cycle analysis. Decompositions of aggregate output into a trend and a cyclical component or into trend, cycle and noise have an intrinsic interest in economic assessments. Moreover, cyclical developments in activity measures, such as GDP or unemployment, play a prominent role in Phillips Curve models that explain inflation dynamics. Finally, the proper analysis of the fiscal policy stance requires estimating cyclically adjusted budget balances.

Local polynomial regression [5-8] is a well-known method in the statistical literature, which has been used for business cycle analysis [9]. The underlying idea of this nonparametric method is that any function can be well approximated by a Taylor series expansion in the neighborhood of any point. This smooth function is then a natural estimator of the low frequency (long-run) component of the series (i.e., the trend or the trend-cycle component). When these methods are adopted for business cycle estimation, estimates of the business cycle are made with the contrast filter obtained by the difference of the series with this low frequency component. The resulting component is therefore obtained by a kind of high-pass filter, so that it provides an estimate of the cycle (plus short-run noise).

The aim of this communication is to assess the validity of local polynomial regression-based methods for business cycle analysis. We contribute to the literature in several ways. First, we provide a frequency domain interpretation of cyclical filters obtained on the basis of standard local polynomial regression methods, taking different assumptions about the bandwidths and kernels functions, and show that they act as a kind of high-pass filters so that they result in noisy cycles. Second, we propose what we call band-pass local polynomial regression, given that they operate as a kind of band-pass filter, as contrasts of cyclical filters and provide their frequency domain interpretation. Third, we compare these estimators to frequently used filters, such as Hodrick-Prescott, Baxter and King and Butterworth filters. Fourth, we illustrate the procedures using US GDP series. 


\section{Materials and Methods}

\subsection{Standard (High-Pass) Local Polynomial Regression}

The aim of standard local polynomial regression is to estimate the trend of a series $y_{t}$ as a possibly nonlinear function $m\left(x_{t}\right)$ of a variable $x_{t}$

$$
y_{t}=m\left(x_{t}\right)+\varepsilon_{t}
$$

where $\varepsilon_{t}$ is an error term and the functional form $m(x)$ does not have a closed-form expression, so that standard nonlinear estimators cannot be applied. Here, we consider that $x_{t}$ is a deterministic variable, specifically a polynomial in time $(t)$. To obtain the trend for a given date $\left(t_{0}\right)$, a regression is fit using only the data belonging to an interval around $t_{0}$. The width of the interval used (the bandwidth) is a fixed number $(h)$. As $h$ gets large, the local polynomial fit approaches the polynomial fit using the whole sample. Specifically,

$$
y_{t}=a\left(t_{0}\right)+b_{1}\left(t_{0}\right)\left(t-t_{0}\right)+\ldots+b_{k}\left(t_{0}\right)\left(t-t_{0}\right)^{k}+\varepsilon_{t} t \in\left[t_{0}-h, t_{0}+h\right] t=h+1, \ldots, n-h
$$

Each of these regressions is fit using weighted least squares (WLS), solving the following minimization problem and the trend estimate is then obtained as the fitted value of the regression.

$$
\min _{a, b} \sum_{t=1}^{n} K\left(\frac{t-t_{0}}{h}\right)\left(\left(y_{t}-a\left(t_{0}\right)-b_{1}\left(t_{0}\right)\left(t-t_{0}\right)+\ldots+b_{k}\left(t_{0}\right)\left(t-t_{0}\right)^{k}\right)^{2}\right.
$$

The trend estimate depends on a number of factors: First, the kernel function $K$, which provides the weights for WLS. In the literature, several kernels have been proposed, the most common of which are shown in Table 1. The different kernels give more weight to points near $t_{0}$ and less weight to points further away, but they differ in the precise weights. Second, the window width $(h)$, which determines the number of points used in each regression. Increasing (decreasing) $h$ involves using a wider (narrower) interval, which tends to increase (decrease) the smoothness of the trend. Third, the degree of the polynomial. In practice, choosing a local linear polynomial seems to provide a good balance between flexibility in reproducing the data and computational ease.

Table 1. Alternative Kernel Functions.

\begin{tabular}{cc}
\hline Kernel Name & Kernel Function \\
\hline Epanechnikov (EP) & $\frac{3}{4}\left(1-u^{2}\right) I(|u| \leq 1)$ \\
Biweight (BI) & $\frac{15}{16}\left(1-u^{2}\right)^{2} I(|u| \leq 1)$ \\
Rectangular (RE) & $\frac{1}{2} I(|u| \leq 1)$ \\
Triangular (TR) & $(1-|u|) I(|u| \leq 1)$ \\
\hline
\end{tabular}

Note: $u$ is the argument of the kernel function and $I(|u| \leq 1)$ is an indicator function that takes a value of one if its argument is true, and zero otherwise.

As noted in [6], the trend estimate of the local polynomial regression is simply a linear function of the dependent variable

$$
\hat{y}_{t}=\sum_{j=-h}^{h} r_{j} y_{t-j}
$$

where $r_{j}$ are weights, that depend on the kernel function, the bandwidth and the degree of the polynomial.

It is easily seen that, in the interior of a time series, the weights implied by the local polynomial regression are shift-invariant, so that the local polynomial fit works like a linear filter in the lag operator 
$\left(L y_{t} \equiv y_{t-1}\right)$. Further, it is easily shown that if the kernel function is symmetric, then the weights are symmetric $\left(r_{-j}=r_{j}\right)$, so the filter can be rewritten as

$$
\Phi(L)=r_{0}+\sum_{j=1}^{h} r_{j}\left(L^{-j}+L^{j}\right)
$$

Note that symmetry of weights implies that the filter has no phase shift, so the timing of turning points is not distorted by this filter.

If $\omega \in[0, \pi]$ denotes the frequency measured in radians, replacing $L$ by the complex number $e^{-i \omega}$ and using the identity $e^{-i j \omega}+e^{i j \omega}=2 \cos (j \omega)$ gives the gain of the trend estimation filter

$$
G_{T}(\omega)=r_{0}+\sum_{j=1}^{h} r_{j}\left(e^{-i \omega j}+e^{i \omega j}\right)=r_{0}+2 \sum_{j=1}^{h} r_{j} \cos (j \omega)
$$

In the context of business cycle estimation, estimates of the business cycle are made with the contrast filter obtained by the difference of the series with its low frequency component. The resulting component is, strictly speaking, a cyclical plus noise component, but in applications [9] is taken as an estimator of the cycle. The gain function of the linear filter that estimates the cycle (plus noise) is

$$
G_{C}(\omega)=1-G_{T}(\omega)
$$

The gain function determines how the different cyclical fluctuations contribute to the signal. If $G_{C}\left(\omega_{0}\right)=1$ cyclical fluctuations with period $p=\frac{2 \pi}{\omega_{0}}$ are fully passed, whereas if $G_{C}\left(\omega_{0}\right)=0$ they are suppressed.

As shown in the results section below, local polynomial regression-based methods for business cycle analysis operate as a kind of high-pass filter, so in the comparisons below we compare them to an ideal high-pass filter. Formally, the ideal high-pass filter $\left(G_{I}^{H P}\right)$ has a gain function given by

$$
G_{I}^{H P} \quad(\omega)= \begin{cases}0 & \text { if } \omega<\omega \\ 1 & \text { if } \omega_{1} \leq \omega\end{cases}
$$

which means that cyclical fluctuations belonging to the interval $\left[\omega_{1}, \pi\right]$ pass through the filter untouched, but all other fluctuations are completely removed. In the empirical literature on the business cycle it is customary to consider 8 years (or 32 quarters) as a cut-off point.

\subsection{Band-Pass Local Polynomial Regression}

As shown in the results section below, local polynomial regression-based methods for business cycle analysis operate as a kind of high-pass filter. In the literature, trend plus cycle plus noise decompositions are often considered; the business cycle corresponds to a range of frequencies in a filter that is bounded both below and above, in order to censor both low frequency trends and high-frequency noise. This can be implemented by means of a band-pass filter. Formally, the ideal band-pass filter $\left(G_{I}^{B P}\right)$ has a gain function given by

$$
G_{I}^{B P} \quad(\omega)=\left\{\begin{array}{rr}
0 & \text { if } \omega<\omega_{1} \\
1 & \text { if } \omega_{1} \leq \omega \leq \omega_{2} \\
0 & \text { if } \omega>\omega_{2}
\end{array}\right.
$$

which means that cyclical fluctuations belonging to the interval $\left[\omega_{1}, \omega_{2}\right]$ pass through the filter untouched, but all other fluctuations are completely removed. In the empirical literature on the business cycle it is customary to consider a band of interest corresponding to fluctuations between 8 and 32 quarters. 
We propose to obtain band-pass local polynomial regression filters by means of the difference of two (high-pass) local polynomial regression-based filters for business cycle analysis.

$$
\Phi^{B P}\left(L ; K_{1}, K_{2}, h_{1}, h_{2}, k_{1}, k_{2}\right)=\Phi^{H P}\left(L ; K_{1}, h_{1}, k_{1}\right)-\Phi^{H P}\left(L ; K_{2}, h_{2}, k_{2}\right)
$$

This filter will depend on two possibly different kernel functions, bandwidths and polynomial degrees. The filtered series would then be obtained as

$$
\hat{y}_{t}^{B P}=\Phi^{B P}(L) y_{t}
$$

For simplicity, below we consider a restricted version of this filter in which the kernel function and polynomial degree are the same. Specifically,

$$
\Phi^{B P}\left(L ; K, h_{1}, h_{2}, k\right)=\Phi^{H P}\left(L ; K, h_{1}, k\right)-\Phi^{H P}\left(L ; K, h_{2}, k\right)
$$

\section{Results}

\subsection{High-Pass Local Polynomial Regression}

Figure 1 presents the implied gain functions of the contrast filter obtained by the difference of the series with its low frequency component using standard linear polynomial regressions to estimate the long run component, considering different kernels and bandwidths and Table 2 the quadratic distances with respect to an ideal high pass filter, focusing on fluctuations with period below 8 years (32 quarters). A comparison of these gain functions to the gain of an ideal high pass show a reasonable performance. Gains vary with the precise kernel and bandwidth chosen -the minimum being given by the Epanichnekov kernel with a bandwidth equal to 15-but there are three qualitative regularities shared by the different variants. First, low frequency movements are removed and high frequency movements fully passed through. Second, low bandwidths result in intermediate cycles being attenuated. Third, gain functions are not monotonically increasing.

Epanechnikov high pass filters Gain as a function of frequency

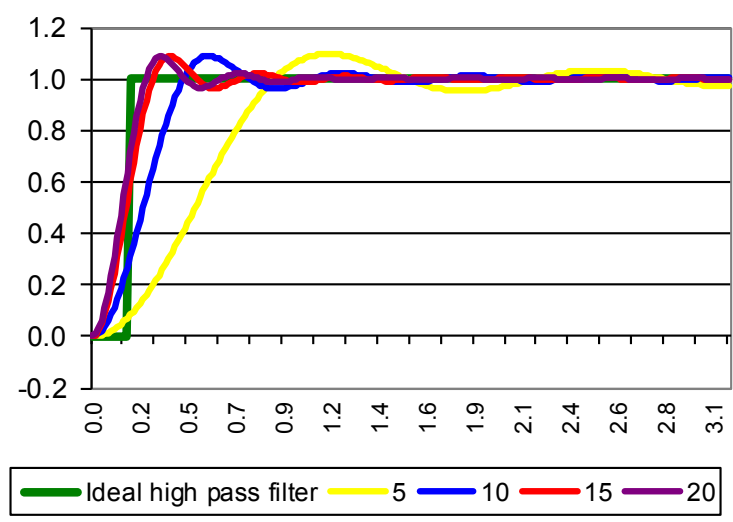

Biweight high pass filters Gain as a function of frequency

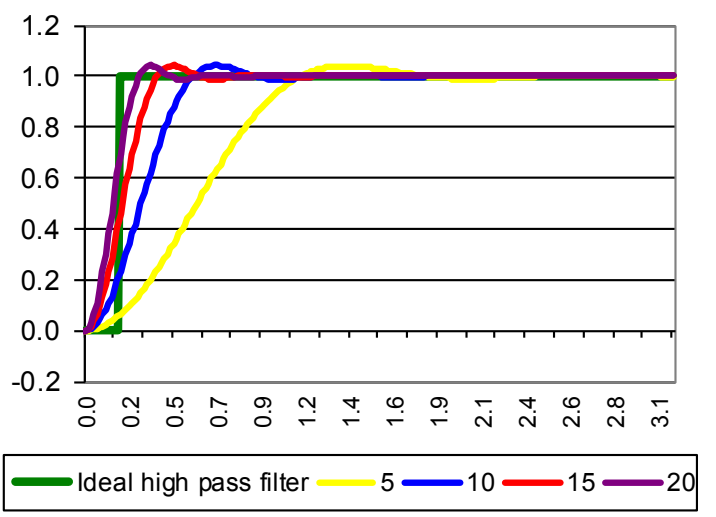

Figure 1. Cont. 
Rectangular high pass filters

Gain as a function of frequency

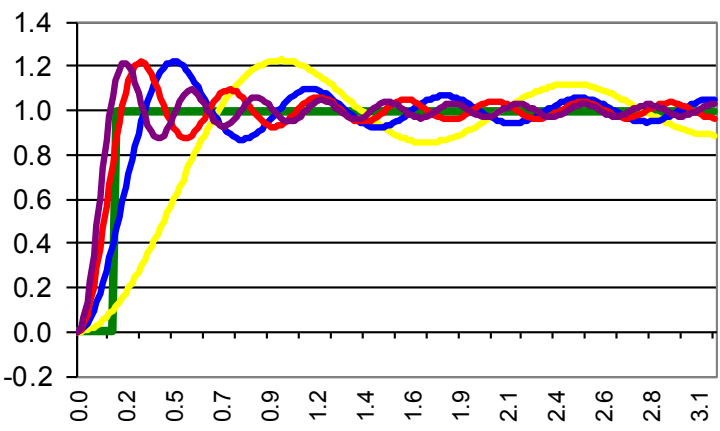

Ideal high pass filter $5 \longrightarrow 10-15-20$
Triangular high pass filters

Gain as a function of frequency

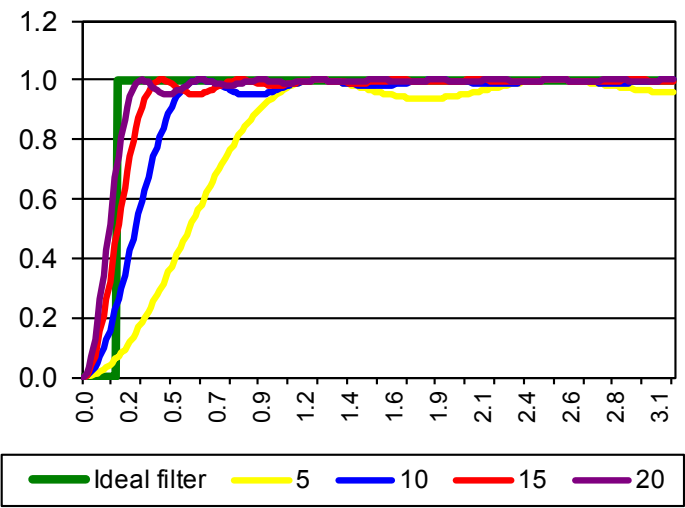

Figure 1. Gain functions of high-pass local polynomial regression filters.

Table 2. Quadratic distances with respect to the ideal high pass filter (multiplied by 100).

\begin{tabular}{ccccc}
\hline Bandwidth & Epanechnikov & Biweight & Rectangular & Triangular \\
\hline 5 & 6.79 & 8.76 & 5.77 & 8.10 \\
10 & 1.43 & 2.24 & 1.23 & 1.92 \\
15 & $\mathbf{0 . 6 6}$ & 0.79 & 1.27 & 0.73 \\
20 & 0.75 & 0.71 & 2.33 & 0.82 \\
\hline
\end{tabular}

Note: Minimum value over kernels and bandwidths in bold font.

\subsection{Band-Pass Local Polynomial Regression}

Figure 2 presents the implied gain functions for band-pass linear polynomial regressions using different kernels and bandwidths. For a given kernel function, the band pass local polynomial regression estimate is defined as the difference between two contrast filters, the high-pass one for a given kernel and bandwidth and the high-pass one with the same kernel and bandwidth ${ }^{1}$ equal to 3 and Table 3 gives the quadratic distances with respect to a band pass filter, focusing on fluctuations with period below 8 years (32 quarters). A comparison of these gain functions to the gain of an ideal high pass show a performance that is not too satisfactory. Gains vary with the precise kernel and bandwidth chosen -the minimum being given by the biweight kernel with a bandwidth equal to 20 minus a biweight kernel with a bandwidth equal to 3- but there are two qualitative regularities shared by the different variants. First, low and high frequency movements tend to be suppressed, as expected from a band pass filter. Second, for a given kernel function the fit is highest for an intermediate value of the bandwidth.

1 Consideration of higher bandwidths resulted in a worse performance. 
Epanechnikov band pass filters Gain as a function of frequency

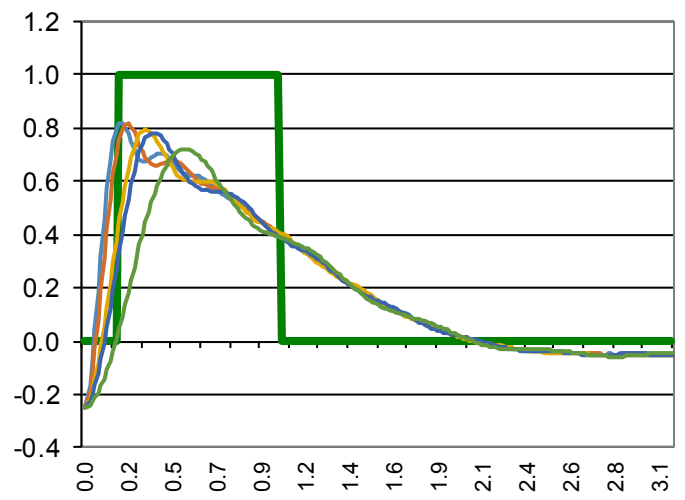

Ideal band pass filter Epanechnikov 30-3

_Epanechnikov 20-3 -Epanechnikov 15-3
- Epanechnikov 10-3

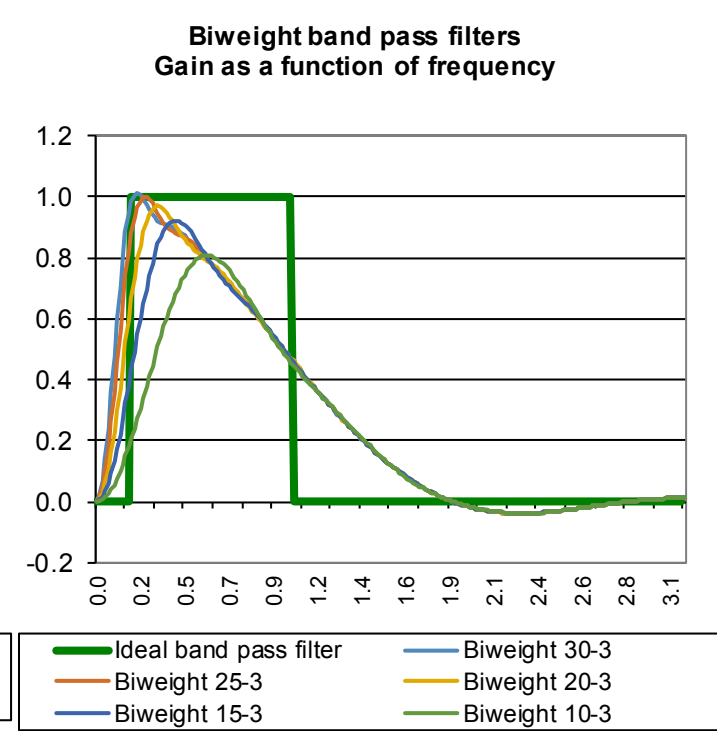

Biweight band pass filters Gain as a function of frequency

Rectangular band pass filters Gain as a function of frequency

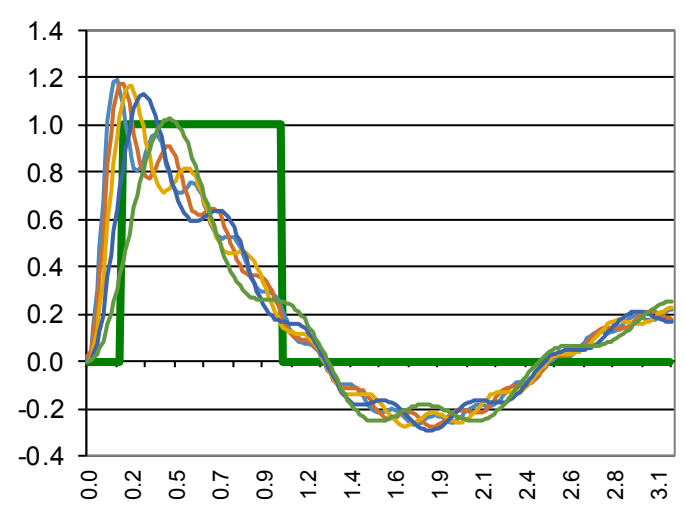

Triangular band pass filters

Gain as a function of frequency

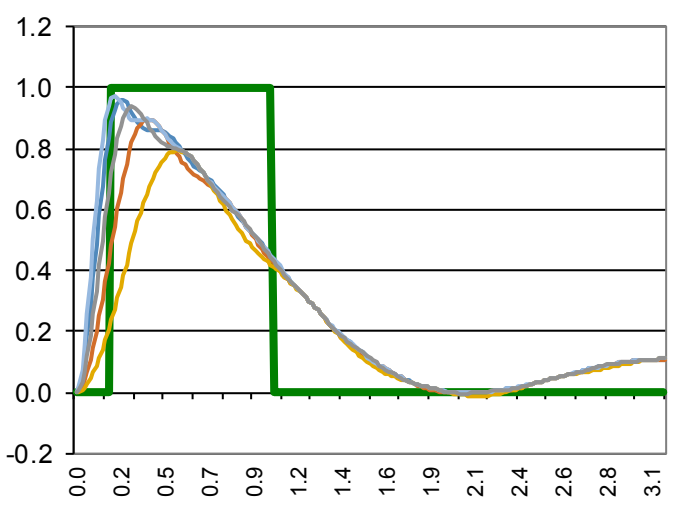

Figure 2. Gain functions of band pass local polynomial regression filters.

Table 3. Quadratic distances with respect to the ideal band pass filter (multiplied by 100).

\begin{tabular}{ccccc}
\hline Bandwidth & Epanechnikov & Biweight & Rectangular & Triangular \\
\hline 5 & 20.38 & 16.70 & 13.05 & 15.54 \\
10 & 9.15 & 6.35 & 8.63 & 6.53 \\
15 & 6.87 & 4.60 & 8.27 & 4.87 \\
20 & 6.69 & 4.46 & 9.08 & 4.82 \\
25 & 6.97 & 4.98 & 10.11 & 5.30 \\
30 & 7.43 & 5.57 & 10.92 & 5.89 \\
\hline
\end{tabular}

Note: Differences with a filter with the same kernel and bandwidth equal to 3 . Minimum value over kernels and bandwidths in bold font.

\subsection{Empirical Applications: US Business Cycles}

In this section, we document some features of aggregate economic fluctuations, which are commonly referred to as business cycles. We consider quarterly data from the postwar US economy. More specifically, we consider the Real Gross Domestic Product seasonally adjusted series, as released by the US Bureau of Economic Analysis. The sample period starts in 1947 Q1 and ends in 2016 Q2. 
We apply local polynomial regression-based methods and band-pass local polynomial regression methods to characterize the US business cycle, considering different kernel functions and bandwidths.

Regarding high-pass local polynomial regressions, we highlight the following results (Figure 3). First, as expected from the gain functions, we find that trends are removed, but noise is still present in what is typically termed the cyclical component. Second, there is an important degree of comovement among the different measures, both in terms of bandwidths and kernel functions. Third, higher bandwidths result in cyclical components with higher standard deviations.
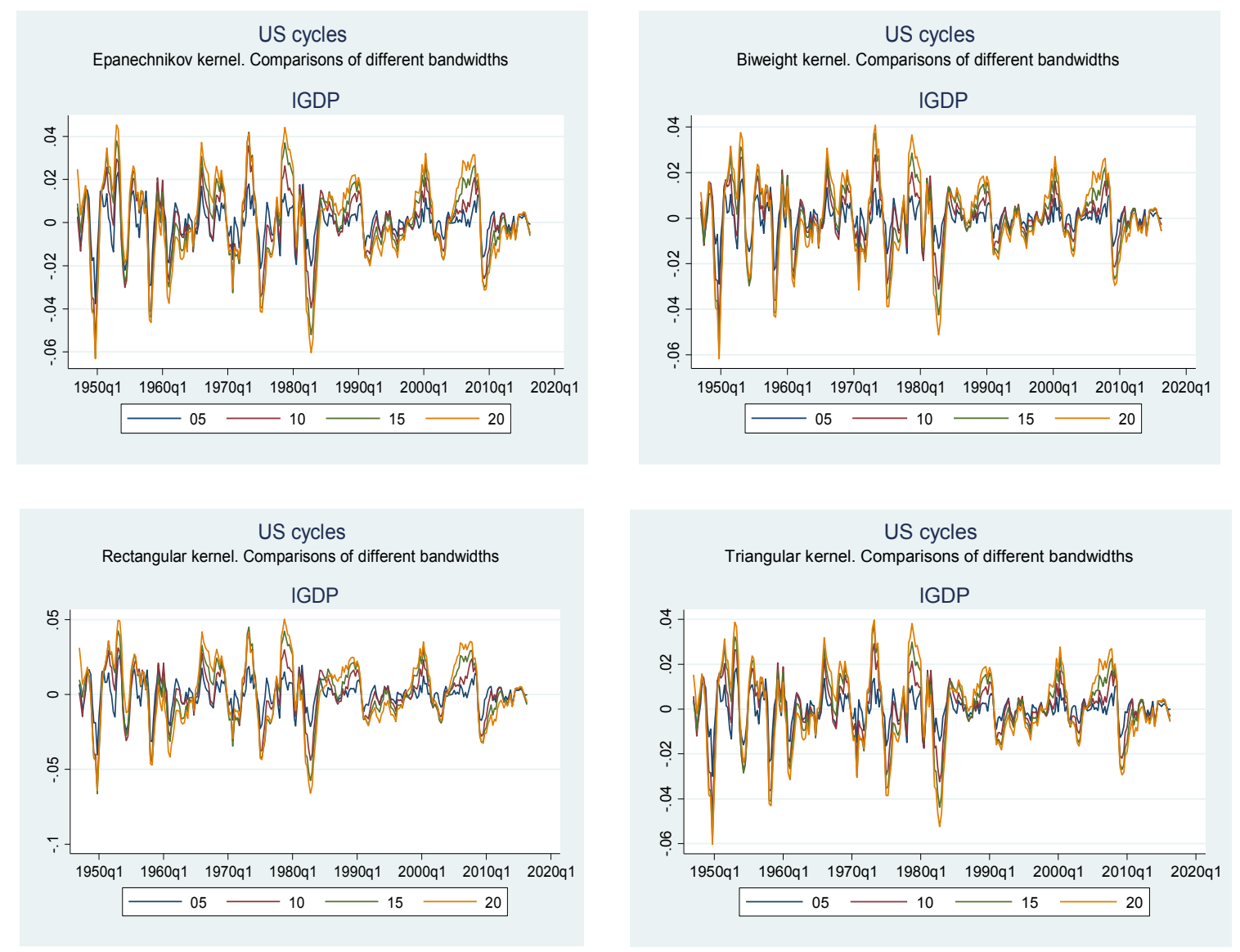

Figure 3. US Business Cycles. High-pass local polynomial regressions.

Regarding band-pass local polynomial regressions, we highlight the following results (Figure 4). First, as expected from the gain functions, we find that trends and noise are removed, so that estimates are smoother than those derived from standard local polynomial regression methods. Second, there is an important degree of comovement among the different measures, both in terms of bandwidths and kernel functions. Third, higher bandwidths result in cyclical components with higher standard deviations. 

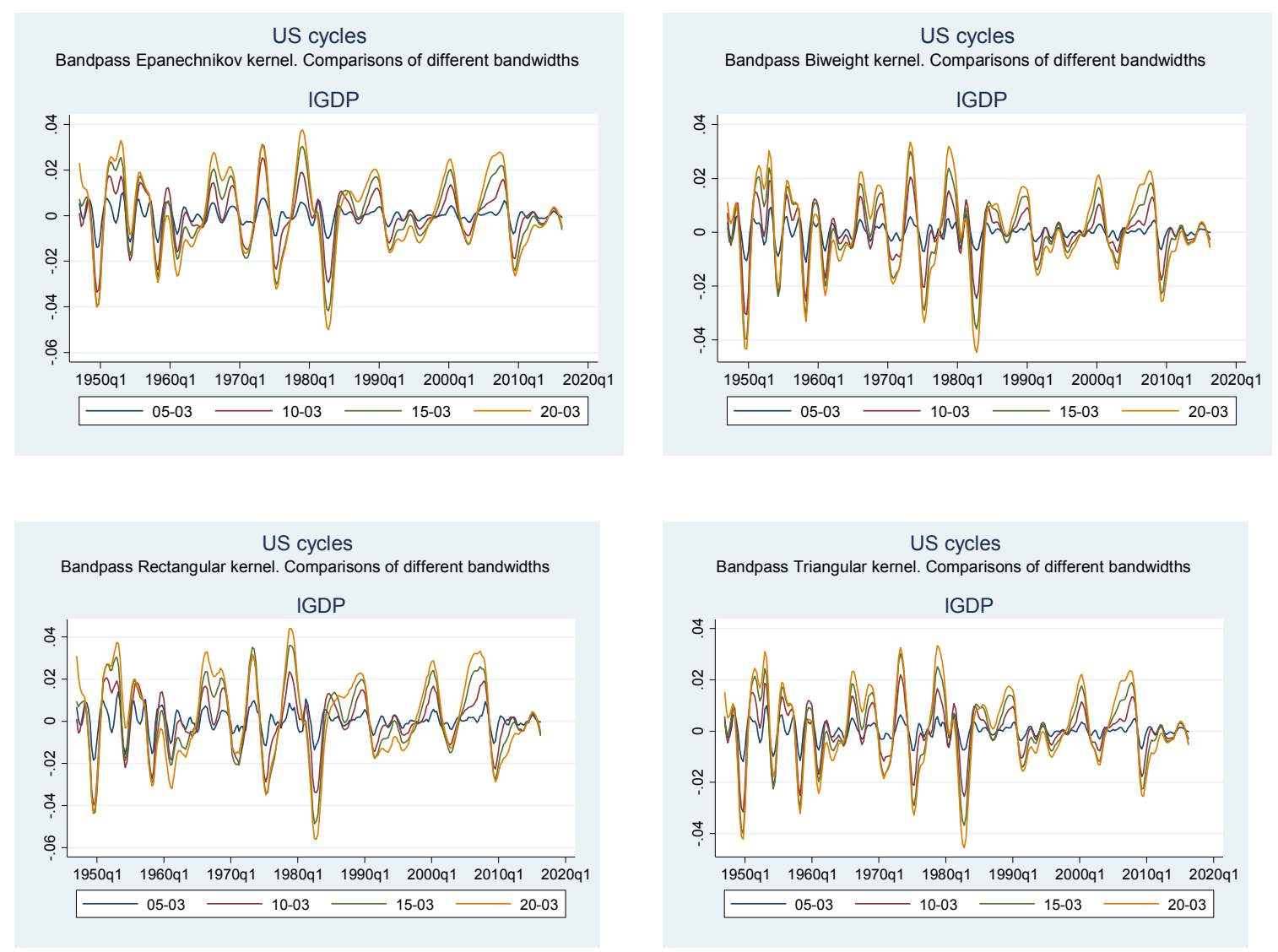

Figure 4. US Business Cycles. Band-pass local polynomial regressions.

\section{Discussion}

\subsection{High-Pass Local Polynomial Regression}

Results of local polynomial regression-based estimators are compared to the widely used Hodrick and Prescott filter [10]. The HP estimator may be considered as a high-pass filter, which damps cyclical fluctuations with high periods and leaves short-run cycles barely untouched. As shown above, this property is also shared by local polynomial regression-based estimators. In Figure 5 and Table 4 we can see that the HP filter with a standard value of lambda equal to 1600 provides a slightly better approximation to the ideal high filter that the best performing local polynomial method (Epanechnikow with bandwidth equal to 15). However, local polynomial-based methods perform better than Hodrick and Prescott filters that use values of the lambda parameter which are higher or lower than the industry standard. It has to be borne in mind that kernel bandwidths are often estimated.

The left panel of Figure 6 compares for the US economy the best performing linear polynomial regression-based method (Epanichnekov with bandwidth equal to 15), with Hodrick Prescott filters. We highlight that for this series the Epanechnikov filter is virtually identical to the standard Hodrick Prescott filter with lambda equal to 1600 . Indeed, their correlation coefficient is 0.99 . We also find that the higher the value of lambda the higher is the variability of the cyclical component. 
High pass filters Gain as a function of frequency

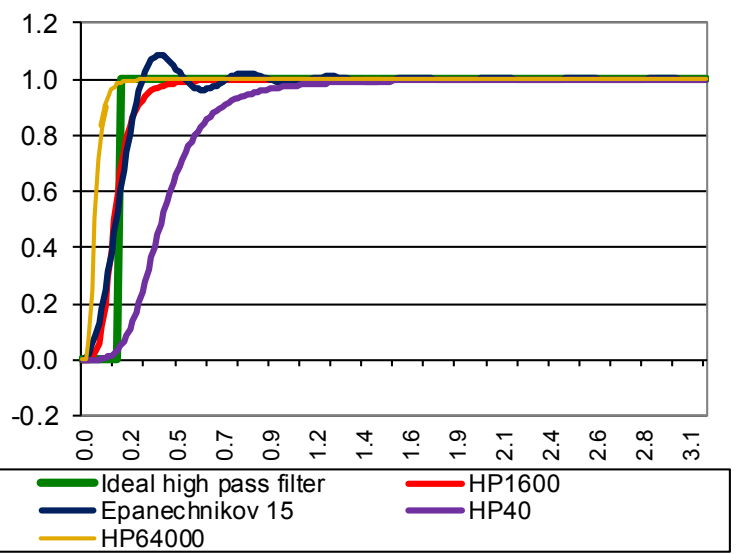

Bandpass filters Gain as a function of frequency

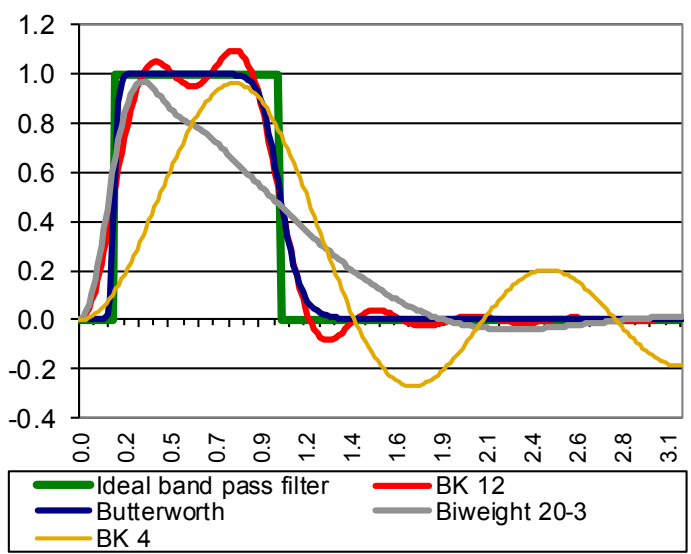

Figure 5. Comparison of different high-pass and bandpass filters.

Table 4. Quadratic distances with respect to the ideal high pass filter (multiplied by 100).

\begin{tabular}{cc}
\hline Lambda & Hodrick-Prescott \\
\hline 40 & 4.91 \\
1600 & $\mathbf{0 . 6 0}$ \\
64,000 & 3.04 \\
\hline
\end{tabular}

Note: Minimum value in bold font.
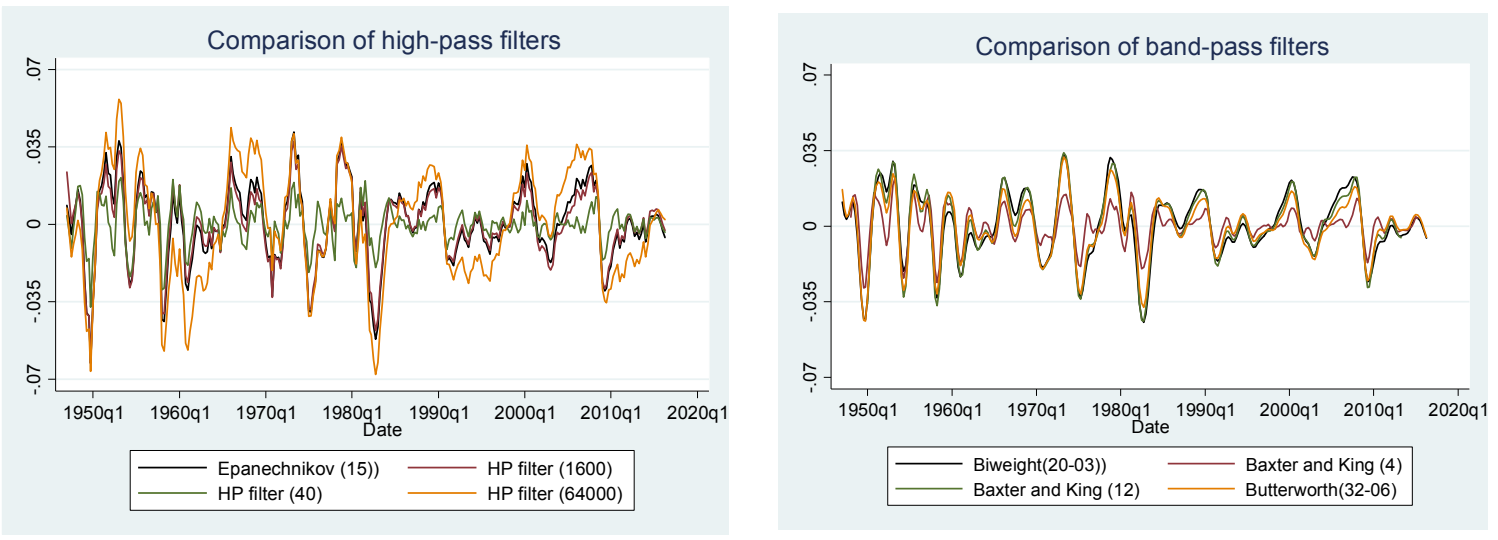

Figure 6. US Business Cycles. Comparison of different high-pass and bandpass filters.

\subsection{Band-Pass Local Polynomial Regression}

Results of band-pass local polynomial estimators are compared to two widely used band pass filters: the filter put forward by Baxter and King [11] and the Butterworth filter [12,13]. In Figure 3 and Table 5 we can see that the best performing band-pass local polynomial regression is closer to the ideal band-pass filter than the one proposed by Baxter and King when the moving average equals 4 [BK(4)]. However, a Butterworth filter or a Baxter and King filter with a moving average equal to 12 $[\mathrm{BK}(12)]$ are closer to the ideal band-pass filter. In quantitative terms, the Butterworth filter provides a much more accurate approximation to the ideal filter than the Baxter and King filter. It has to be borne in mind that kernel bandwidths are often estimated and that better results could be obtained by combining kernels from different families. 
Table 5. Quadratic distances with respect to the ideal band pass filter (multiplied by 100).

\begin{tabular}{ccc}
\hline Baxter \& King $k=\mathbf{4}$ & Baxter \& King $k=\mathbf{1 2}$ & Butterworth \\
\hline 8.64 & 1.41 & $\mathbf{0 . 7 6}$ \\
\hline \multicolumn{3}{c}{ Note: Minimum value in bold font. }
\end{tabular}

The right hand panel of Figure 6 compares for the US economy the best performing band pass local polynomial regression (Biweight 20-03) with band-pass methods typically used in the literature, namely Baxter and King and Butterworth filters. We highlight several results. First, we note that, as expected, band-pass filters provide smoother signals of the cyclical component than typical high-pass filters. Second, we find that the band-pass local polynomial regression closely resembles the Baxter and King (12) filter and Butterworth filters. Indeed, the correlation coefficient is 0.98 with the Baxter and King (12) filter and 0.95 with the Butterworth filter. We note that the Baxter and King filter has a truncation parameter that involves losing estimates of the cyclical component for the first and last periods of the sample. Indeed, Baxter and King (4) loses one year and Baxter and King (12) 3 years of data.

This paper provides a frequency domain interpretation of cyclical components obtained by local polynomial regression-based methods for business cycle analysis. We propose what we call band-pass local polynomial regression, as contrasts of standard local polynomial regression estimates and provide their frequency domain interpretation. We also compare results to frequently used filters, such as Hodrick-Prescott, Baxter and King and Butterworth filters. Procedures and comparisons presented are illustrated using the US GDP series.

Acknowledgments: Alberto Cabrero co-authored a previous version of the paper. Ana Gómez-Loscos provided valuable comments.

Author Contributions: Luis J. Álvarez designed and wrote the paper.

Conflicts of Interest: The author declares no conflict of interest.

\section{References}

1. Canova, F. Detrending and business cycle facts. J. Monet. Econ. 1998, 41, 475-512. [CrossRef]

2. Mills, T.C. Modelling Trends and Cycles in Economic Time Series; Palgrave Macmillan: Basingstoke, UK, 2003.

3. Dagum, E.B. A cascade linear filter to reduce revisions and false turning points for real time trend-cycle estimation. Econom. Rev. 2009, 28, 40-59. [CrossRef]

4. Pollock, D.S.G. Econometric filters. Comput. Econ. 2016, 48, 669-691. [CrossRef]

5. Stone, C.J. Consistent nonparametric regression. Ann. Stat. 1977, 5, 595-620. [CrossRef]

6. Cleveland, W.S. Robust locally weighted regression and smoothing scatterplots. J. Am. Stat. Assoc. 1979, 74, 829-836. [CrossRef]

7. Fan, J.; Gijbels, I. Local Polynomial Modelling and Its Applications: Monographs on Statistics and Applied Probability 66; CRC Press: Boca Raton, FL, USA, 1996.

8. Pollock, D.S.G. Handbook of Time Series Analysis, Signal Processing, and Dynamics; Academic Press: Cambridge, MA, USA, 1999.

9. Leamer, E.E. Housing is the Business Cycle. NBER Working Paper Series 13428; National Bureau of Economic Research: Cambridge, MA, USA, 2007.

10. Hodrick, R.J.; Prescott, E.C. Postwar U.S. Business cycles: An empirical investigation. J. Money Credit Bank. 1997, 29, 1-16. [CrossRef] 
11. Baxter, M.; King, R.G. Measuring business cycles approximate band-pass filters for economic time series. Rev. Econ. Stat. 1999, 81, 575-593. [CrossRef]

12. Butterworth, S. On the theory of filter amplifiers. Exp. Wirel. Wirel. Eng. 1930, 7, 536-541.

13. Gómez, V. The use of Butterworth filters for trend and cycle estimation in economic time series. J. Bus. Econ. Stat. 2001, 19, 365-373. 